www.jmscr.igmpublication.org

Impact Factor (SJIF): 6.379

Index Copernicus Value: 71.58

ISSN (e)-2347-176x ISSN (p) 2455-0450

crossref DOI: https://dx.doi.org/10.18535/jmscr/v6i5.100

Journal Of Medical Science And Clinical Research

IGM Publication

An Official Publication of IGM Publication

\title{
Psychiatric Co-Morbidities in Patients Presenting with Headache: A Prospective Study
}

\author{
Authors \\ Dr K. Balamurali ${ }^{1}$, Dr K.V. Prabhu ${ }^{2}$, Dr M. Thangaraj ${ }^{3}$, Dr I. Meenakshi ${ }^{4}$, \\ Dr M. Arun Kumar ${ }^{5}$ \\ ${ }^{1}$ Assistant Professor, ${ }^{3}$ Professor and HOD, ${ }^{5}$ DM Neurology Resident \\ Department of Neurology, Govt. Thanjavur Medical College, Thanjavur \\ ${ }^{2}$ Resident, ${ }^{4} \mathrm{Head}$ of the Department, Department of Psychiatry, Govt. Thanjavur Medical College, Thanjavur \\ Corresponding Author \\ Dr K.V. Prabhu
}

Resident, Department of Psychiatry, Govt. Thanjavur Medical College, Thanjavur, India.

\begin{abstract}
Background: Psychiatric disorders are commonly encountered in patients attending neurology OPD and headache is one of the most common complaints addressed by primary care physicians and neurologists. These psychiatric comorbidities are likely to be missed if proper evaluation of these patients is not done. Psychiatric co-morbidities worsen the headache and there is a vicious cycle of headache and psychiatric symptoms aggravating each other. It is of utmost importance that an early psychiatric consultation is sought so that this vicious cycle can be broken. This study is conducted to find out the prevalence of psychiatric co-morbidity among patients attending neurology OPD for various types of headache.

Aims and Objectives: To study the prevalence of psychiatric co-morbidity in various types of headaches in the outpatient Department of Neurology in a tertiary care hospital.

Materials and Methods: This was a prospective cohort study in which all the Patients presenting with primary complaints of headache were included on the basis of a predefined inclusion and exclusion criteria. The type of headache was diagnosed by a Neurologist. Basic investigations like complete blood count, Blood sugar level, estimation of resting blood pressure was done in all patients. These basic investigations were followed by CT Brain and EEG. All patients underwent psychiatric consultation to confirm or rule out Presence of psychiatric comorbidities and to undergo the necessary psychological evaluation for the diagnosis. The data was tabulated and statistical analysis was done using SPSS 16.0 version software

Results: Out of the 60 subjects with primary complaints of headache there were 21 (35\%) males and 39 (65\%) females with a M:F ratio of 1:1.85. Out of the studied cases 47 patients (78.33\%) had some or the other form of psychiatric co-morbidity. Most common psychiatric co-morbidity was found to be depression which was seen in 18 patients (38.3\%) followed by somatoform disorder (31.9\%), dysthymia (6.38\%), panic disorder (10.63\%), psychogenic non epileptic seizures (8.51\%) and substance dependence (4.25\%). Out of the 60 subjects, 27 patients had Migraine (45\%), 24 patients had Tension type of headache (40\%), 4 patients had Trigeminal Neuralgia (6.67\%), 2 patients had Cluster headache (3.33\%), and 3 patients with headache not classifiable (5\%). Patients with age less than 35 years most commonly had migraine and with age more than 35 years had depression and somatoform disorder, and most of them were females in both groups.

Conclusion: Psychiatric disorders are a significant comorbidity among patients presenting with headache. It is important to identify presence of such co-morbidity so that a timely psychiatric consultation is sought. Such a psychiatric consultation and further psychiatric treatment, if required, is crucial in these patients.

Keywords: Headache, Psychiatric co-morbidity, vicious cycle, management.
\end{abstract}




\section{Introduction}

Headache may arise from pain-sensitive structures within or outside cranium. These pain-sensitive structures include structures such as the skin, muscles, blood vessels and nerves (outside cranium) or large intracranial arteries or veins, dura and dural vessels, and cranial nerves (intracranial) ${ }^{1}$. On the other hand, the structures like brain parenchyma, ependymal lining of the ventricles and choroid plexus are all pain insensitive. Headache may broadly be divided into three broad categories that include vascular (Migraine, cluster headache and toxic vascular headache), myogenic (myositis, psychogenic and cervical spine arthritis) and traction headaches (intracranial mass lesions, cerebrovascular diseases and inflammatory conditions) ${ }^{2}$. Headache is one of the most common symptoms for which patients attend or are referred to neurology OPD. It is said that headache as a symptom occurs almost in $99 \%$ of the individuals at some or the other stage of their lives. The one-year prevalence of headache in individuals is found to be almost $90 \%$. Although a vast majority of headaches are benign and not associated with any significant pathology it may evoke a great amount of concern and anxiety in patients as well as their relatives or caregivers $^{3}$. It however must be emphasized that headache may be first symptom of more sinister pathologies like intracranial space occupying lesions, hydrocephalus and intracranial infections ${ }^{4}$. Therefore, a systematic history directed to find out the probable etiologic factor producing the headache is the treating neurologist's most valuable tool that may help in arriving at a specific diagnosis ${ }^{5}$. Primary headache is defined as one which is not associated with any structural, metabolic or other lesion in the body in general and in brain particularly ${ }^{6}$. Despite not being associated with any serious structural or metabolic disorder primary headache may cause significant disability with reduced efficiency, quality of life and loss of work days ${ }^{7}$. The association of headache with psychiatric comorbidity is well known since more than a century but recently this association has gained immense interest amongst researchers. Many studies and randomized trials have found psychiatric co-morbidity in migraine and tension type headaches in approximately $80-85 \%$ of the patients. Whether headache aggravates psychiatric symptoms or psychiatric symptoms cause headache has been subject of many researches. Irrespective of whether these psychiatric symptoms are cause or effect of headache it is important to understand that psychiatric symptoms aggravates headache and headache causes psychiatric symptoms setting up a vicious cycle ${ }^{9}$. This vicious cycle needs to be interrupted by proper evaluation and management of psychiatric comorbidity in patients with headache. Various psychiatric co-morbidities seen in patients presenting primarily for headache may include Depression, Suicidal thoughts, worthlessness, hopelessness, affective and anxiety disorders, panic attacks and phobic disorders. In many patient chronic headache is accompanied by disturbed sleep which again may aggravate headache and resolution of this disturbed sleep once again is crucial for successful management of these patients. Recognition and treatment of psychiatric co-morbidity in patients with chronic and recurrent headaches is necessary to improve quality of life, prognosis and reduce the risk of chronicity of the disease ${ }^{10}$. Keeping in mind above facts we undertook this prospective cohort study to estimate the prevalence of psychiatric comorbidity in various types of primary headache and the factors influencing it.

\section{Materials and Methods}

This was a prospective study of 60 patients conducted at a tertiary care medical institute situate in an urban area. Institutional ethical committee approved the study. All the patients with primary complaints of headache attending neurology OPD were included in this study on the basis of a predefined inclusion criteria. Any patient having any exclusion criteria was excluded from the study. Informed consent was taken from 
all the patients. A detailed history was taken. sociodemographic profile of the patient was noted down. The analysis of type of primary headache in terms of location, onset, duration, associated factors, aggravating and relieving factors, current medications and their duration was done. All patients were subjected to detailed clinical and neurological examination. Imaging in the form of CT brain in all patients and MRI in selected patients (in whom CT findings were equivocal). Patients with any organic intracranial lesions were excluded from the study. Complete blood count and Erythrocyte Sedimentation Rate was done in all the patients to rule out temporal arteritis and if positive were excluded from the study. After being evaluated for the type of primary headache, they were asked for specific psychiatric complaints, previous history of psychiatric treatment, family history of psychiatric illness, history of substance of abuse usage like alcohol, smoking etc. and were subjected to psychological evaluation using specific rating scales like Hamilton's Rating scale for anxiety and depression, Young mania rating scale etc., and psychiatric analysis after duly applying ICD-10 diagnostic criteria for depression, bipolar disorder, panic disorder, somatoform disorder, substance dependence syndrome and DSM-V criteria for diagnosing dysthymia and psychogenic non epileptic seizures. The results were studied using appropriate statistical methods. Data analysis was carried out using SPSS16.0 version software. Microsoft word and excel were used for generating charts and graphs.

\section{Inclusion Criteria}

1- Patients attending neurology OPD with a primary complaint of headache.

2- Age more than 18 years.

3- Informed Consent was given by patients.

\section{Exclusion Criteria}

1. Age less than 18 years.

2. Those who refused informed consent.

3. Patients with organic brain diseases.

4. Patients with temporal arteritis.

\section{Results}

Out of the 60 subjects presenting to neurology OPD for the primary complaint of headache there were $21(35 \%)$ males and $39(65 \%)$ females with a M: F ratio of $1: 1.85$.

\section{Gender Distribution}

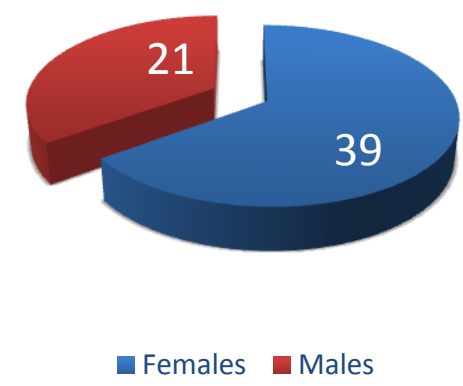

Figure 1: Gender Distribution of the studied cases.

The analysis of affected age group showed that the most common age group presenting with primary complaint of headache was $20-40$ years $(60 \%)$ followed by 41-60 years $(30 \%)$. Age groups of less than 20 years and more than 60 years were equally affected at each formed $5 \%$ of the total patients.

Table 1: Age groups of the studied cases

\begin{tabular}{|l|c|c|}
\hline Age group & No of patients & Percentage \\
\hline$<\mathbf{2 0}$ years & 3 & $5 \%$ \\
\hline $\mathbf{2 0 - 4 0}$ years & 36 & $60 \%$ \\
\hline $\mathbf{4 1 - 6 0}$ years & 18 & $30 \%$ \\
\hline $\mathbf{> 6 0}$ years & 3 & $5 \%$ \\
\hline
\end{tabular}

Most of them belonged to low socio economic status and educated less than $8^{\text {th }}$ standard. Most of them were farmers from rural areas. $75 \%$ of the studied cases were married and were living with spouses. $20 \%$ patients were unmarried and $5 \%$ patients were married but either widowed or separated. $30 \%$ of them were in joint family and majority of them (70\%) were living in a nuclear family set up. 
Table 2: Demographic characteristics of the studied cases

\begin{tabular}{|c|c|c|c|}
\hline Demographic Factors & Sociodemographic variable & $\mathbf{N}$ & $\%$ \\
\hline \multirow{3}{*}{$\begin{array}{l}\text { Family Income } \\
\text { (Per Month) }\end{array}$} & Less than 5000 & 30 & $50 \%$ \\
\hline & $5000-10000$ & 18 & $30 \%$ \\
\hline & More than 10000 & 12 & $20 \%$ \\
\hline \multirow{4}{*}{ Educational Status } & Illiterate & 30 & $50 \%$ \\
\hline & Upto $8^{\text {th }}$ Standard & 14 & $23.3 \%$ \\
\hline & Higher Secondary & 10 & $16.7 \%$ \\
\hline & Graduate and Above & 6 & $10 \%$ \\
\hline \multirow{2}{*}{ Residence } & Rural & 49 & $81.7 \%$ \\
\hline & Urban & 11 & $18.3 \%$ \\
\hline \multirow{3}{*}{ Marital Status } & Married & 45 & $75 \%$ \\
\hline & Unmarried & 12 & $20 \%$ \\
\hline & Lost a spouse & 3 & $5 \%$ \\
\hline \multirow[t]{2}{*}{ Type of Family } & Joint & 18 & $30 \%$ \\
\hline & Nuclear & 42 & $70 \%$ \\
\hline \multirow[t]{4}{*}{ Occupation } & Agriculture & 40 & $66.7 \%$ \\
\hline & Employed/salaried & 12 & $20 \%$ \\
\hline & Unemployed & 3 & $13.3 \%$ \\
\hline & Own small business & 5 & $8.33 \%$ \\
\hline
\end{tabular}

Out of the 60 subjects, 27 patients had Neuralgia (6.67\%), 2 patients had Cluster Migraine (45\%), 24 patients had Tension type headache (3.33\%) and 3 patients with headache of headache $(40 \%)$, 4patients had Trigeminal not classifiable $(5 \%)$.

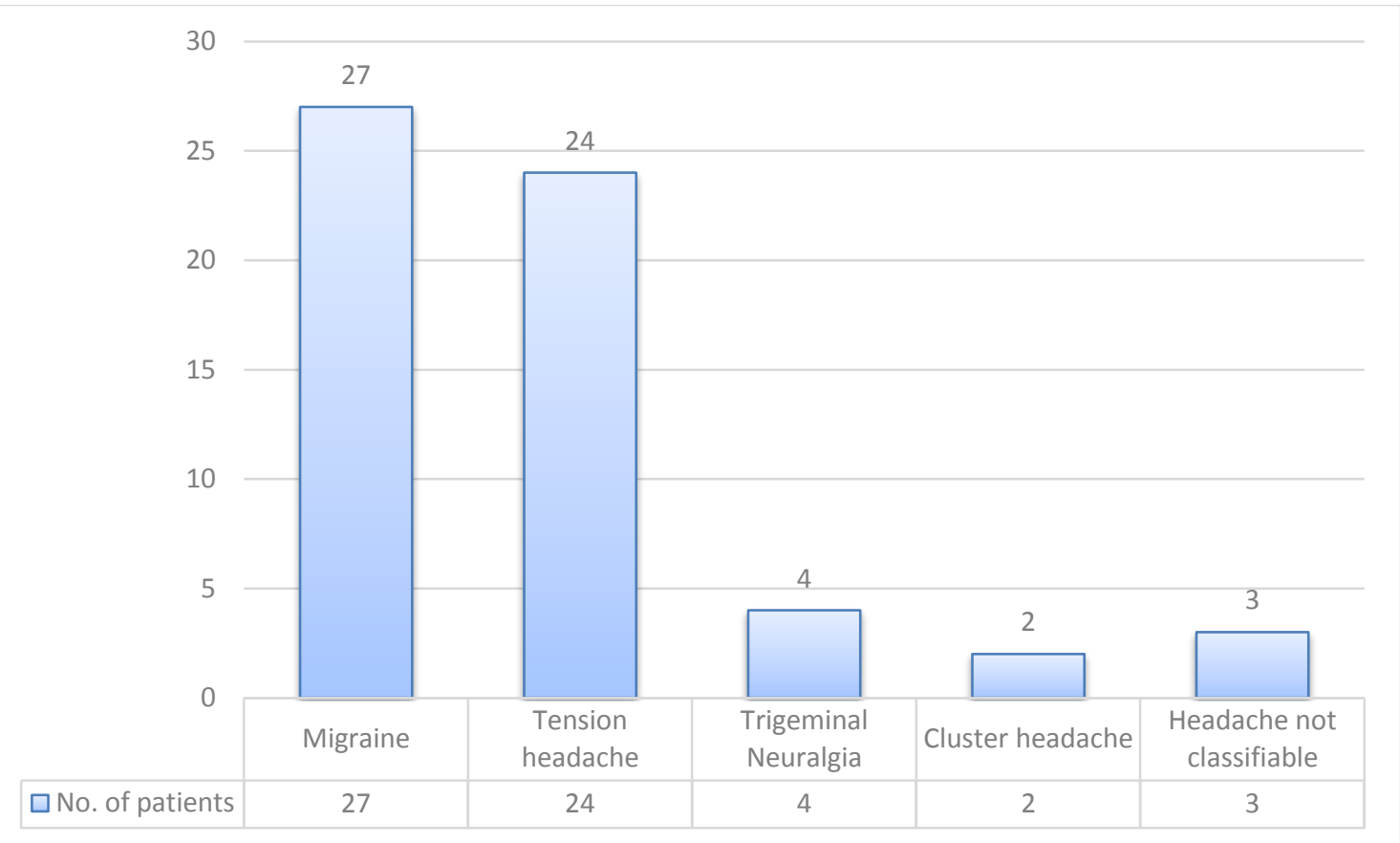

Fig 2 : Break up of Neurological diagnosis in studied cases

Out of the 60 subjects with primary complaints of headache 47 subjects $(78.33 \%)$ had psychiatric co-morbidity. The most common psychiatric comorbidity in the studied cases was found to be depression which was seen in 18 patients
(38.3\%) (mild-5, moderate-10, severe-3) followed by somatoform disorder $(31.9 \%)$, dysthymia $(6.38 \%)$, panic disorder $(10.63 \%)$, psychogenic non epileptic seizures $(8.51 \%)$ and substance dependence $(4.25 \%)$. 


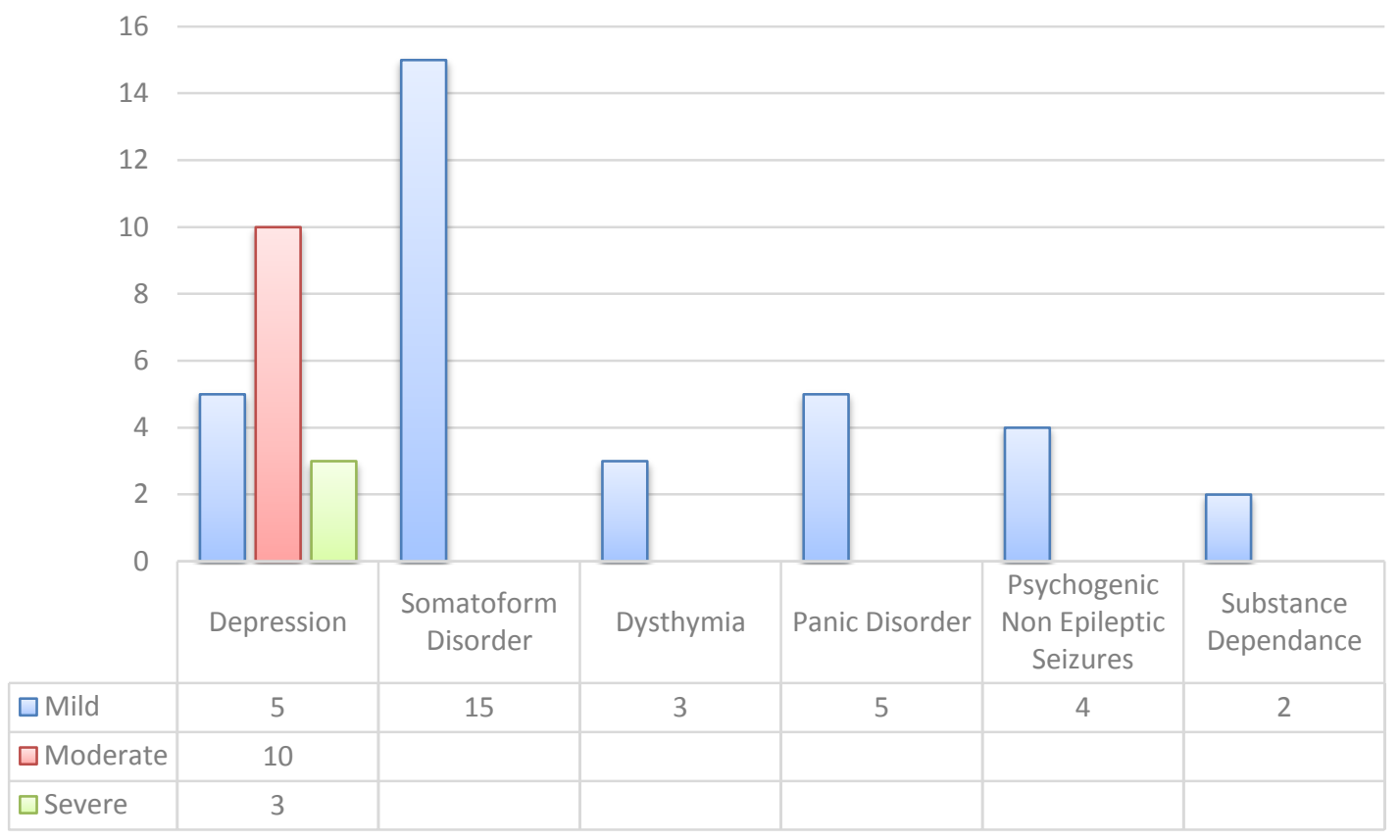

Figure 3: Breakup of Psychiatric Diagnosis in studied cases

The analysis of patients with psychiatric comorbidity showed that dysthymia and Somatoform disorder were common among patients with Migraine. Depression and Panic disorders were common among patients with tension type of headache. Out of 4 patients with psychogenic non epileptic seizures, 3 patients were with headache unclassifiable, and 1 patient had tension type of headache. Married females with an average age of 35 with agriculture as their prime occupation presenting with migraine type of headache were having more somatoform disorders whereas Males with an average age of 40 with agriculture as their prime occupation presenting with tension type of headache were having more affective disorders and panic disorders. The common presenting complaints in the studied cases after headache were found to be body ache $(55 \%)$, disturbed sleep (53.3\%) and fatigue (46.6\%).

\section{Presenting complaints}

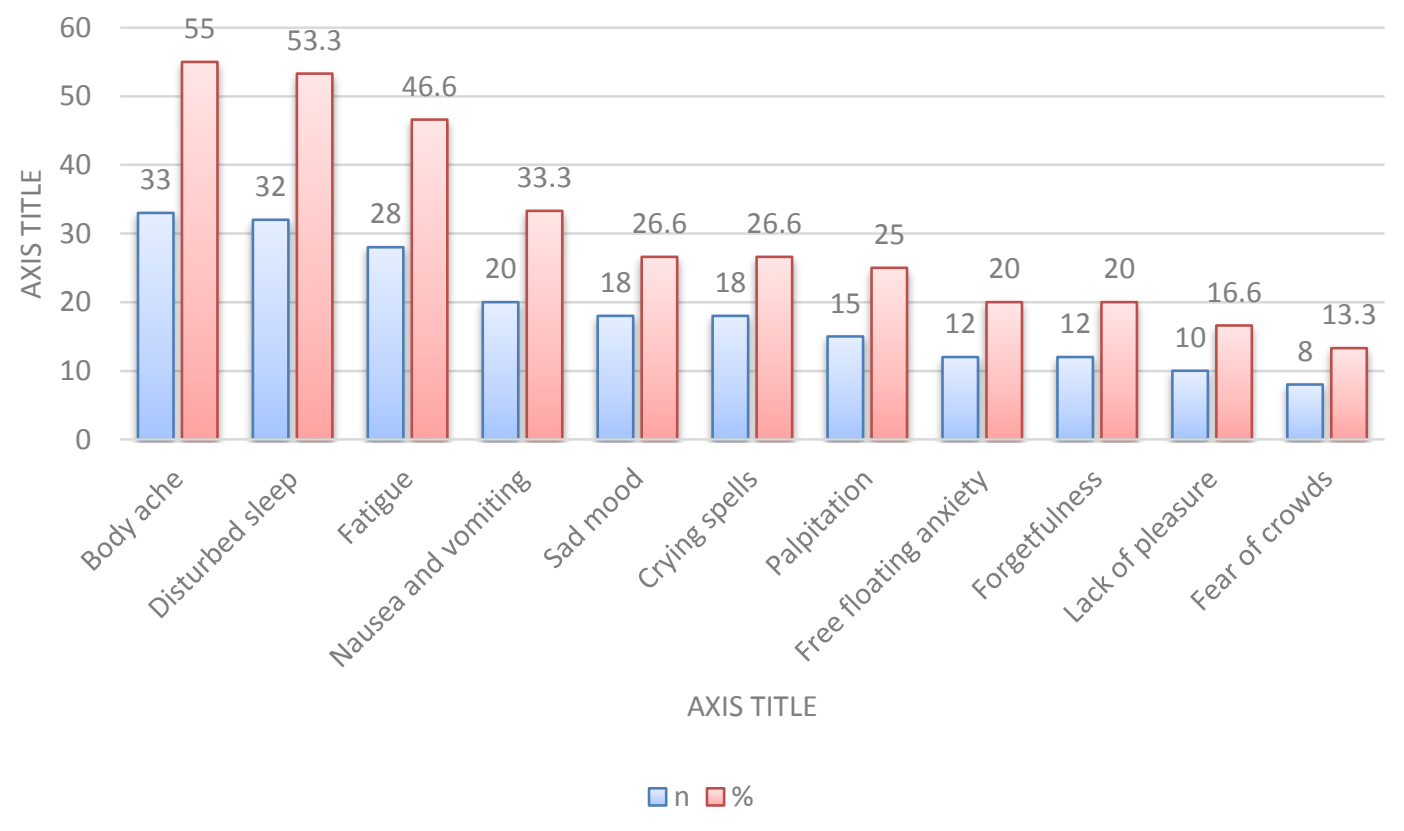

Figure 4 : Presenting complaints, in addition to headache, in studied cases 


\section{Discussion}

It was not until the end of the nineteenth century that Freud categorically associated the concepts of psychopathology with common place migraine. Wolff has been credited with developing the influential notion of the 'the migraine personality' that he characterized as a medley of 'personality features and reactions dominant in individuals with migraine', including 'feelings of insecurity with tension manifested as inflexibility, conscientiousness, meticulousness, perfectionism, and resentment ${ }^{11}$. Numerous epidemiological studies have revealed that psychiatric disorders (e.g. depression and anxiety) occur with greater frequency among recurrent headache patients than among the general population ${ }^{12}$.

In this study, out of 60 cases, 47 patients $(78.33 \%)$ had psychiatric co-morbidity according to ICD-10 classification. Among these 21 were men (35\%) and 39 were women $(65 \%)$. A large number of women patients, especially middle-aged ones, had migraine as well as depression. Longitudinal data indicate that relative to men, women are fourtimes more likely to develop migraine and twotimes more likely to develop major depression. 54 subjects (90\%) were between 20 and 60 years of age. Most of the subjects with migraine had onset during early 30 s while those with tension headache had a middle age onset; they were mostly women. Thirty subjects (50\%) were earning less than Rs.5000 and were more susceptible to onset of headache as well as psychiatric morbidity. Thirty subjects $(18 \%)$ were illiterate, $12(20 \%)$ were unmarried and 3 persons (5\%) had lost a spouse; $42(70 \%)$ had a nuclear family, 3 (13.3\%) were unemployed. Family history of mental illness and past history of mental illness was present in $10(16.67 \%)$ and $6(10 \%)$ subjects, respectively. They were all susceptible to headache and depression.

The comorbidity reported by Alvin et al. ${ }^{13}$ is as follows: MDD (34\%), dysthymia (9\%), bipolar II $(4 \%)$, manic episode $(5 \%)$, panic disorder $(11 \%)$, generalized anxiety disorder (GAD) (10\%), obsessive-compulsive disorder (OCD) (9\%), phobia (40\%), illicit drug use (20\%), and nicotine dependence (33\%). In comparison, this study shows MDD (30\%), dysthymia (5\%), panic disorder $(8.33 \%)$, psychogenic non epileptic seizures $(6.7 \%)$, somatoform disorder $(25 \%)$, and substance dependence $(3.33 \%)$. The incidence of MDD, dysthymia, panic disorder is comparable, but the results of this study report no GAD, OCD or bipolar disorder, surprisingly this study found more somatoform disorder \& psychogenic non epileptic seizures and less substance dependence $^{14}$.

Wacogne et al. measured the intensity of stress, anxiety and depression in a sample of 141 patients with migraine compared with a control group of 109 non-migraine workers matched for age and sex. Their results indicated that stress and anxiety were higher in the migraine group than in the control group. The main symptoms were 'morning fatigue', 'intrusive thoughts about work', 'feeling under pressure', 'impatience', and 'irritability'. In the present study, disturbed sleep $(53.3 \%)$, free floating anxiety $(20 \%)$, sad mood $(26.6 \%)$, lack of pleasure (16.6\%), body ache (55\%) and fatigue $(46.6 \%)$ were the main complaints ${ }^{15}$.

Headache may be a form of 'somatization' (a term used for the pathology, e.g. depression) when patients cannot verbalize their mental symptoms but present them by way of somatic symptoms. It was also called 'depressive equivalent' and was considered a typical manifestation of depression in non-industrialized countries ${ }^{16,17}$. This hypothesis has been challenged by Patel who proved that this phenomenon is also common in industrialized countries ${ }^{18}$. This may be a crosscultural phenomenon. However, there is at least some evidence that headache can be a manifestation of a somatoform disorder. The most common somatoform disorder associated with headache was 'undifferentiated somatoform disorder'. In somatoform disorder, headache would represent only one of many medically unexplained somatic complaints such as fatigue, loss of appetite, gastrointestinal symptoms, and urinary complaints. 
Recent characterizations of psychopathology and head- ache have implicated shared neuropathic mechanisms between migraine and affective disorders and bidirectional influences. Both concepts refer to neuro plasticity processes in cortico-limbic structures, where an expanding cortico-limbic field becomes activated by both nociceptors and psychological stimuli over a period of time, resulting in an integrated relationship between migraine (or pain) and psychiatric disturbance in susceptible individuals ${ }^{19}$.

Evidence suggests that patients with elevated psychological symptoms are more likely to seek medical assistance. When present, psychiatric comorbidity often complicates management of headache and portends a poorer prognosis for treatment of headache. These results indicate that patients with long history and high frequency of headaches might benefit from psychiatric evaluation ${ }^{20}$.It is important that neurologists and primary care physicians be sensitized to look for psychiatric symptoms in patients presenting with headache.

\section{Conclusions}

Headache was the main somatic presentation of psychiatric morbidity in nearly $80 \%$ of subjects in this study. The associated psychiatric morbidity included depression, dysthymia, panic disorder, somatoform disorder, psychogenic non epileptic seizures and substance abuse.

Middle-aged women with migraine, people who were illiterate, unemployed, or had lost a spouse and with a family and past history of mental illness were more likely to develop mental illness. Disturbed sleep, free floating anxiety, sad mood, lack of pleasure, body ache and fatigue were the main presenting complaints along with headache.

\section{Limitations}

This study comprised of small sample size. Better planned, longitudinal studies are required to study this area further.

\section{Conflict of Interest: None}

\section{References}

1. Ahmed F. Headache disorders: differentiating and managing the common subtypes. British Journal of Pain. 2012;6(3):124-132.

2. Headache Classification Committee of the International Headache Society (IHS). The International Classification of Headache Disorders, 3rd edition (beta version). Cephalalgia. 2013 Jul;33(9):629-808.

3. Song T-J, Cho S-J, Kim W-J, Yang KI, Yun C-H, Chu MK. Anxiety and Depression in Tension-Type Headache: A Population-Based Study. Fuh J-L, ed. PLoS ONE. 2016;11(10):e0165316.

4. Kristoffersen ES, Lundqvist C, Aaseth K, Grande RB, Russell MB. Management of secondary chronic headache in the general population: the Akershus study of chronic headache. The Journal of Headache and Pain. 2013;14(1):5.

5. Maizels M. The clinician's approach to the management of headache. Western Journal of Medicine. 1998;168(3):203-212.

6. Friedman BW, Grosberg BM. Diagnosis and management of the primary headache disorders in the emergency department setting. Emergency medicine clinics of North America. 2009;27(1):71-viii.

7. Cavallini A, Micieli G, Bussone G, Rossi F, Nappi G. Headache and quality of life. Headache. 1995 Jan;35(1):29-35.

8. Erratum in: Headache 1995 Jun;35(6):314. Guidetti V, Galli F, Fabrizi P, Giannantoni AS, Napoli L, Bruni O, Trillo S. Headache and psychiatric comorbidity: clinical aspects and outcome in an 8-year,followup study. Cephalalgia. 1998 Sep;18(7): 455-62.

9. Torelli P, Lambru G, Manzoni GC. Psychiatric comorbidity and headache: clinical and therapeutical aspects. Neurol Sci. 2006 May;27 Suppl 2:S73-6.

10. Desai SD, Pandya RH. Study of psychiatric comorbidity in patients with 
headache using a short structured clinical interview in a rural neurology clinic in Western India. Journal of Neurosciences in Rural Practice. 2014;5(Suppl 1):S39-S42.

11. Breslau N, Davis GC. Migraine, physical h ealth and psychiatric,disorder: A prospecti ve epidemiologic study in young adults. J Psychiatr Res 1993;27:211

12. Merikangas KR, Angst J, Isler H. Migraine and psychopathology: Results of Zurich cohort study of young adults. Arsch Gen Psychiatry 1990;47:849-53.

13. Alvin E, Lake III, Jeanetta CR, et al. Head ache and psychiatric comorbidity:

Historical context, clinical implications and research relevance. Headache: The Journal of Head and Face Pain 2005; 45:493-506.

14. Radat F, Swendsen J. Psychiatric comorbidity in migraine: A review. Cephalalgia 2005;25:165-78.

15. Wacogne C, Lacoste JP, Guillibert E, et al. Stress, anxiety, depression and migraine. Cephalalgia 2003;23:451-5.

16. Chaturvedi SK, Micheal A, Sarmakadam S. Somatization and psychiatric care. Indian J Psychiatry 1987;29:337-42.

17. Lloyd GA. Psychiatric syndrome with somatic presentation. J Psychosom Res 1986;113-20.

18. Patel V. Cultural factors and international epidemiology. Br Med Bull 2001;57:3345.

19. Merikangas KR, Stevens DE. Comorbidity of migraine and psychiatric disorders. Neurol Clin. 1997 Feb;15(1):115-23.

20. Mitsikostas DD, Thomas AM. Comorbidity of headache and depressive disorders. Cephalalgia 1999;19:211. 